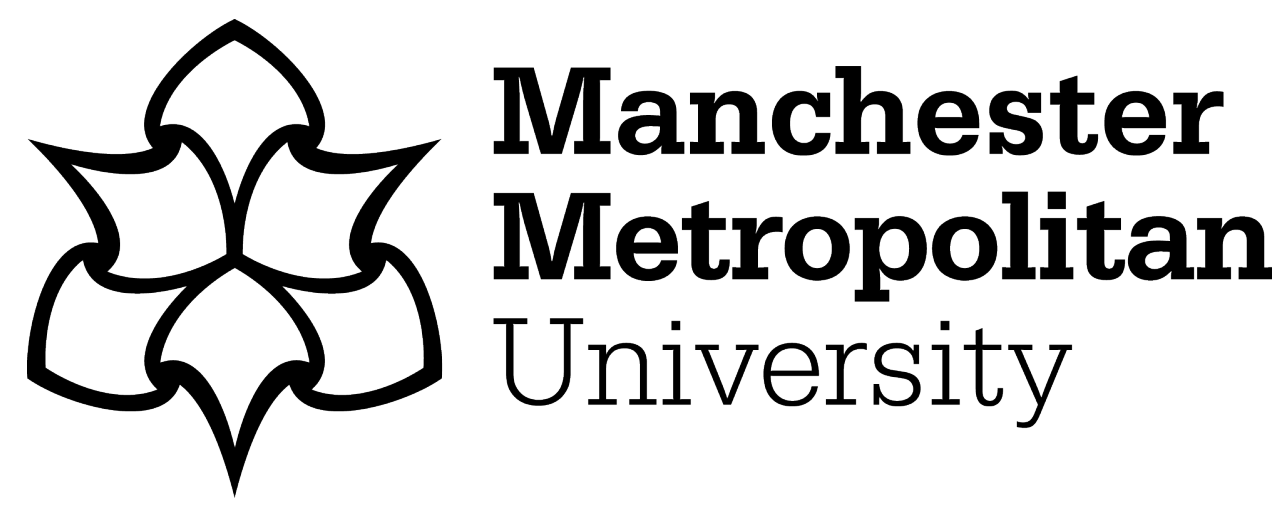

Gire, N, Chaudhry, IB, Naeem, F, Duxbury, J, Riley, M, McKeown, M, Taylor, CD, Taylor, PJ, Emsley, R, Caton, N, Kelly, J, Kingdon, D and Husain, N (2016) TechCare: Mobile-assessment and therapy for psychosis: An intervention for clients within the early intervention service. European Psychiatry, 33 Sup. S608-S608. ISSN 0924-9338

Downloaded from: https://e-space.mmu.ac.uk/621248/

Version: Accepted Version

Publisher: Elsevier

DOI: https://doi.org/10.1016/j.eurpsy.2016.01.2274

Please cite the published version 


\section{TechCare: Mobile-assessment and therapy for psychosis: An intervention for clients within the early intervention service}

N. Gire1,*, I.B. Chaudhry2, F. Naeem3, J. Duxbury1, M. Riley 4, M. McKeown1, C.D. Taylor5, P.J. Taylor6, R. Emsley7, N. Caton4, J. Kelly 4, D. Kingdon8, N. Husain2

1 University of Central Lancashire, School of Health, Preston, United Kingdom

2 The University of Manchester, Institute of Brain Behaviour and

Mental Health, Manchester, United Kingdom

3 Queens University, Psychiatry, Kingston, Canada

4 Lancashire Care NHS Foundation Trust, Early Intervention Service,

Preston, United Kingdom

5 The University of Manchester, School of Psychology, Manchester,

United Kingdom

6 University of Liverpool, Institute of Psychology, Health \& Society,

Liverpool, United Kingdom

7 The University of Manchester, Institute of Population Health,

Manchester, United Kingdom

8 University of Southampton, Psychiatry, Southampton, United

Kingdom

* Corresponding author.

Introduction In the UK, mental illness is a major source of disease burden costing in the region of $£ 105$ billion pounds. mHealth is a novel and emerging field in psychiatric and psychological care for the treatment of mental health difficulties such as psychosis. Objective To develop an intelligent real-time therapy (iRTT) mobile intervention (TechCare) which assesses participant's symptoms in real-time and responds with a personalised self-help based psychological intervention, with the aim of reducing participant's symptoms. The system will utilise intelligence at two levels:

- intelligently increasing the frequency of assessment notifications if low mood/paranoia is detected;

- an intelligent machine learning algorithm which provides interventions in real-time and also provides recommendations on the most popular selected interventions.

Aim The aim of the current project is to develop a mobile phone intervention for people with psychosis, and to conduct a feasibility study of the TechCare App.Methods The study consists of both qualitative and quantitative components. The study will be run across three strands:

- qualitative work;

- test run and intervention refinement;

- feasibility trial.

Results Preliminary analysis of qualitative data from Strand 2 (test run and intervention refinement) in-depth interviews with service users $(n=2)$ and focus group with health professionals $(n=1)$, highlighted main themes around security of the device, multimedia and the acceptability of psychological interventions being delivered via the TechCare App.

Conclusions Research in this area can be potentially helpful in addressing the demand on mental health services globally, particularly improving access to psychological interventions. Disclosure of interest The authors have not supplied their declaration of competing interest. 